\title{
Comparação e análise do uso de revisão sistemática e revisão de escopo na área do cuidado ao paciente na Farmácia
}

Comparison and analysis of the use of systematic review and scoping review in the area of patient care in Pharmacy

Comparación y análisis del uso de la revisión sistemática y revisión del alcance en el área de atención al paciente en Farmacia

Recebido: 26/08/2021 | Revisado: 01/09/2021 | Aceito: 09/09/2021 | Publicado: 12/09/2021

Tatiane Perusse Coelho

ORCID: https://orcid.org/0000-0003-4093-197X Universidade Federal de Minas Gerais, Brasil

E-mail: perussetati@gmail.com

Cristiane de Paula Rezende

ORCID: https://orcid.org/0000-0001-7457-4187 Universidade Federal de Minas Gerais, Brasil E-mail: cris7paula@gmail.com

Maria do Carmo Vilas Boas Sousa ORCID: https://orcid.org/0000-0001-8779-8323 Universidade Federal de Minas Gerais, Brasil E-mail: mariavilassboas@gmail.com

Carlos Eduardo de Oliveira Pereira ORCID: https://orcid.org/0000-0002-7250-6110 Universidade Federal de Minas Gerais, Brasil E-mail: carloseduardo.farmacia@gmail.com

Simone de Araújo Medina Mendonça ORCID: https://orcid.org/0000-0001-5792-0682 Universidade Federal de Minas Gerais, Brasil E-mail: simoneamm@ufmg.br

\begin{abstract}
Resumo
O presente estudo tem como objetivo descrever e comparar dois métodos de revisão - sistemática e de escopo - além de analisar o uso destes métodos em estudos na área do cuidado ao paciente na farmácia. Os artigos utilizados para fundamentar este trabalho foram selecionados nas bases de dados: Google scholar, PubMed e Scielo. O estudo foi dividido em duas etapas: 1) Descrição e comparação de dois métodos de revisão da literatura: revisão sistemática e a revisão de escopo; 2) Análise e comparação do uso de revisões de escopo e sistemática na área de cuidado ao paciente na Farmácia. Constatou-se que os métodos para a elaboração das revisões estudadas seguem um processo sistemático e bem estruturado, mas se diferem quanto a alguns elementos metodológicos não observados na revisão de escopo, como a avaliação da qualidade dos estudos incluídos e análises estatísticas mais avançadas. A revisão sistemática analisada, ao contrário da revisão de escopo, não atendeu aos critérios propostos para a sua elaboração nas etapas de seleção e avaliação da qualidade dos estudos. Conclui-se que, embora as revisões se destinem a objetivos diferentes, ambas apresentam uma metodologia criteriosa. Associar corretamente a pergunta de pesquisa com o tipo de revisão é crucial para a condução do estudo.
\end{abstract}

Palavras-chave: Revisão de Escopo; Revisão Sistemática; Estudo Comparativo; Assistência ao Paciente; Serviços farmacêuticos.

\begin{abstract}
This study aims to describe and compare two review methods - systematic and scoping - in addition to analyzing the use of these methods in studies in the area of patient care in pharmacy. The articles used to support this work were selected from the following databases: Google scholar, PubMed, and Scielo. The study was divided into two stages: 1) Description and comparison of two literature review methods: systematic review and scoping review; 2) Analysis and comparison of the use of scoping and systematic reviews in the area of patient care in Pharmacy. It was found that the methods for preparing the studied reviews follow a systematic and well-structured process, but they differ in terms of some methodological elements not observed in the scoping review, such as quality assessment of included studies and more advanced statistical analyses. The systematic review analyzed, unlike the scoping review, did not meet the criteria proposed for its preparation in the stages of selection and evaluation of the quality of studies. It is concluded that, although the reviews are aimed at different objectives, both present a careful methodology. Correctly associating the research question with the type of review is crucial for conducting the study.
\end{abstract}


Keywords: Scoping Review; Systematic review; Comparative study; Patient care; Pharmaceutical services.

\section{Resumen}

Este estudio tiene como objetivo describir y comparar dos métodos de revisión, sistemático y de alcance, además de analizar el uso de estos métodos en estudios en el área de atención al paciente en farmacia. Los artículos utilizados para apoyar este trabajo fueron seleccionados de las siguientes bases de datos: Google Scholar, PubMed y Scielo. El estudio se dividió en dos etapas: 1) Descripción y comparación de dos métodos de revisión de la literatura: revisión sistemática y revisión del alcance; 2) Análisis y comparación del uso del alcance y revisiones sistemáticas en el área de atención al paciente en Farmacia. Se encontró que los métodos para preparar las revisiones estudiadas siguen un proceso sistemático y bien estructurado, pero difieren en términos de algunos elementos metodológicos no observados en la revisión del alcance, como la evaluación de la calidad de los estudios incluidos y análisis estadísticos más avanzados. La revisión sistemática analizada, a diferencia de la revisión de alcance, no cumplió con los criterios propuestos para su elaboración en las etapas de selección y evaluación de la calidad de los estudios. Se concluye que, si bien las revisiones están dirigidas a objetivos diferentes, ambas presentan una metodología cuidada. Asociar correctamente la pregunta de investigación con el tipo de revisión es crucial para realizar el estudio.

Palabras clave: Revisión del alcance; Revisión sistemática; Estudio comparativo; Asistencia al paciente; Servicios farmaceuticos.

\section{Introdução}

O crescente volume de estudos publicados favoreceu o desenvolvimento de diferentes abordagens de sistematização de achados da literatura (Cordeiro \& Soares, 2019). Nesse contexto, estudos de revisão são essenciais para o desenvolvimento científico e sua solidez fundamenta e orienta a prática da saúde baseada em evidências (SBE) (Mancini \& Sampaio, 2006).

A SBE é uma conduta que visa solucionar divergências para a tomada de decisão, uma vez que reúne os melhores e mais recentes achados acerca do tema proposto (Mendes \& Silveira, 2008). Essa conduta fornece embasamento científico para o cuidado em saúde, inclusive norteia a prática do cuidado farmacêutico. Durante a prestação do serviço clínico, o farmacêutico deve se fundamentar nas melhores evidências científicas disponíveis para tomar decisão em farmacoterapia. Sendo assim, a confiabilidade das informações consultadas é de suma importância para garantir a qualidade do cuidado prestado (Comissão de Farmácia Comunitária do Estado do Pará, 2005; Mancini \& Sampaio, 2006; Mendes \& Silveira, 2008).

Diante disso, métodos sistemáticos são utilizados para evitar viés e viabilizar uma avaliação mais objetiva dos resultados analisados, propiciando uma síntese clara e objetiva a respeito de uma intervenção ou tema (Sampaio \& Mancini, 2007). Um dos métodos mais difundidos na área acadêmica é a revisão sistemática. Esse método sumariza as evidências provenientes de diferentes estudos em torno de um tema específico, mediante a aplicação de métodos explícitos e sistematizados de busca, além da apreciação crítica e síntese das informações encontradas (Sampaio \& Mancini, 2007). Outro método de revisão de literatura proposto inicialmente por Arksey e O'Malley (2005) é a revisão de escopo. Esse método também apresenta uma metodologia sistemática e bem estruturada, sendo comumente utilizado para mapear a literatura existente em um determinado campo em termos de sua natureza, características e volume, além de, esclarecer as definições de trabalho e os limites conceituais de um tópico ou área (Peters \& Godfrey, 2015).

Apesar de existirem várias publicações disponíveis na literatura que orientam como elaborar cada uma dessas revisões (Cordeiro \& Soares, 2019; Sampaio \& Mancini, 2007; Hedges \& Cooper, 1994; Aromataris \& Munn 2020a; Peters et al., 2020), ainda ocorrem dúvidas a respeito de suas metodologias e aplicações. Isso pode justificar o fato de, em muitos casos, o tipo de revisão escolhida pelo pesquisador não ser o mais indicado, e/ou o padrão metodológico preconizado na literatura não ter sido adotado adequadamente, invalidando, assim, a confiabilidade científica da revisão (Mancini \& Sampaio, 2006).

Mediante o exposto, o presente artigo teve como objetivo descrever e comparar dois métodos de revisão de literatura a revisão sistemática e a revisão de escopo - além de analisar o uso destes métodos em estudos na área do cuidado farmacêutico. 


\section{Metodologia}

Este trabalho foi estruturado de acordo com Köche (2011).

A elaboração deste estudo foi dividida em duas etapas:

\section{Etapa 1 - Descrição e comparação de dois métodos de revisão da literatura: revisão sistemática e a revisão de escopo}

Para fundamentar a descrição e comparação entre as revisões, foi realizada uma busca sistemática nas bases de dados Google scholar, PubMed e Scielo, no período entre novembro de 2019 a maio de 2020, por meio da seguinte estratégia de busca: (metodologia OR methodology OR elaboração OR elaborat* OR condução OR orientação OR diretriz OR guideline OR guid*) AND (definição OR definition OR descrição OR description) AND (revisão sistemática OR systematic review) and (revisão de escopo OR scoping review). Além disso, foi realizada busca manual para identificar diretrizes e manuais que orientem como essas revisões devem ser elaboradas.

\section{Etapa 2 - Comparação e análise do uso de revisões de escopo e sistemática na área do cuidado ao paciente na farmácia}

Nesta etapa, os autores do presente estudo optaram por selecionar dois artigos - uma revisão sistemática e uma revisão de escopo - que dizem respeito à mesma temática para viabilizar a comparação dos aspectos metodológicos utilizados entre as revisões. Para isso, partiu-se de uma revisão de escopo (Sousa et al., 2020) desenvolvida anteriormente por duas coautoras deste estudo, que aborda sobre ferramentas para a seleção de pacientes para atendimento farmacêutico. Em seguida, foi realizada uma busca na literatura para identificar a revisão sistemática que seria comparada à revisão de Sousa e colaboradores (2020).

A busca sistemática foi realizada na base de dados PubMed, por meio da seguinte estratégia de busca: (instrumento OR ferramenta OR tools OR measure* OR instrument*) AND (priorizar OR priorit* OR triage* OR acuity) AND (cuidado farmacêutico OR pharmaceutical service OR pharmaceutical care OR comprehensive medication management service) AND (systematic review OR revisão sistemática). Os termos utilizados na estratégia condizem com a temática da revisão de escopo citada anteriormente (Sousa e colaboradores, 2020).

Depois de identificar a revisão sistemática na temática requerida, foi realizada a comparação entre as revisões por meio do uso de duas diretrizes internacionais. A diretriz intitulada Preferred Reporting Items for Systematic Reviews and Meta-Analyses (PRISMA) (Liberati et al., 2009), que consiste em um checklist que contém itens essenciais para conduzir a redação de uma revisão sistemática. E a extensão do checklist PRISMA elaborada para conduzir as revisões de escopo, o PRISMA ScR (Tricco et al., 2018).

\section{Resultados e Discussão}

\section{Etapa 1 - Descrição e comparação de dois métodos de revisão da literatura: revisão sistemática e a revisão de escopo}

Em primeiro lugar, para realizar qualquer tipo de revisão, o pesquisador precisa definir a pergunta de pesquisa. Esta determina o foco da pesquisa, além de nortear as próximas etapas e contribuir no desenvolvimento do protocolo de revisão. No protocolo de revisão, são descritas de forma clara e transparente, todas as etapas do processo que envolve a realização da revisão de literatura, além de definir as análises que serão realizadas (Buehler, Figueiró, Cavalcanti \& Berwanger, 2012). O registro desse protocolo permite que outros pesquisadores contribuam com o autor do estudo fornecendo dados que auxiliem a responder à questão de pesquisa; que outros revisores não iniciem revisões que busquem responder a mesma pergunta, evitando duplicidade de publicações, plágio e o desperdício de energia na análise de artigos que já podem ter sido selecionados; e que as revisões finalizadas e publicadas sejam facilmente identificadas (Barbosa et al., 2019). As bases de dados para o registro das revisões são distintas, sendo a The Cochrane Library e o International prospective register of 
systematic reviews (PROSPERO) opções para registro de revisão sistemática e as bases Open Science Framework e Figshare para registro de revisão de escopo.

A seguir, estão descritas e detalhadas as etapas que devem ser realizadas durante o desenvolvimento das revisões sistemática ou de escopo. Em seguida, são apresentadas as semelhanças e diferenças entre estes tipos de revisão. Para isso, os autores do presente trabalho consultaram o Instituto Joanna Briggs (JBI) para sínteses de evidências (Aromataris \& Munn 2020a; Aromataris \& Munn 2020b; Peters et al., 2020), as diretrizes PRISMA (Liberati et al., 2009), PRISMA-ScR (Tricco et al., 2018) e os artigos encontrados na busca bibliográfica (Cordeiro \& Soares, 2019; Buehler et al., 2012; Suzumura et al., 2014; Buehler, Figueiró, Cavalcanti \& Berwanger, 2014; Donato \& Donato, 2019; Levac, Colquhoun, \& O'Brien, 2010).

\section{Etapas preconizadas para conduzir uma revisão sistemática}

Ao conduzir uma revisão sistemática, o pesquisador precisa ter sua pergunta de pesquisa claramente formulada, além de adotar métodos sistemáticos e explícitos desde a busca na literatura até a análise dos achados dos estudos incluídos. A análise e a sumarização dos resultados dos estudos incluídos podem ou não utilizar métodos estatísticos (Cordeiro, Oliveira, Rentería, Guimarães \& Gers-Rio, 2007). Em suma, todas as etapas preconizadas para elaborar uma revisão sistemática estão elencadas em um checklist de 27 itens denominado Preferred Reporting Items for Systematic Reviews and Meta-Analyses (PRISMA) (Liberati et al., 2009).

\section{Definição da pergunta de pesquisa}

Ter clareza da pergunta de pesquisa é fundamental para manter o foco na temática que a revisão propõe investigar, além de direcionar a condução da revisão e viabilizar uma resposta com menor possibilidade de tendenciosidades (Barbosa et al., 2019; Aromataris \& Munn 2020a). Para contribuir na elaboração de uma pergunta de pesquisa, normalmente, os pesquisadores utilizam acrônimos, que podem ser adaptados para auxiliar em diferentes tipos de pesquisa (Biruel \& Pinto, 2011; B Buehler et al., 2012; Stern, Jordan, \& McArthur, 2014). Na maioria das vezes, revisões sistemáticas que reúnem estudos quantitativos usam os acrônimos PICO, que representa população, intervenção e controle, respectivamente (Buehler $e t$ al., 2012), PECO que simboliza população, exposição, controle e desfecho, respectivamente (Suzumura et al., 2014) ou PIRO que retrata população, teste índice, padrão de referência e desfecho, respectivamente (Buehler et al., 2014). Tais acrônimos são utilizados para estruturar perguntas de pesquisa envolvendo efeitos de uma intervenção, exposição ou que examinam estudos de acurácia, respectivamente. Também existem acrônimos específicos para revisões que reúnem estudos qualitativos (Stern $e t$ al., 2014). Ademais, para revisões cujas perguntas apresentam caráter mais exploratório utiliza-se o acrônimo PVO, que corresponde à população, variável do estudo e desfecho, respectivamente (Biruel \& Pinto, 2011). Para a revisão de escopo é utilizado o acrônimo PCC para população, conceito e contexto (Peters et al., 2020).

\section{Definição dos critérios de elegibilidade e de inclusão}

Os critérios para identificar e selecionar os artigos originais são conhecidos como critérios de elegibilidade (Barbosa et al., 2019; Aromataris \& Munn 2020a). Enquanto, os critérios de inclusão são aqueles que se enquadram no acrônimo e consequentemente, na pergunta norteadora da pesquisa (Buehler et al., 2012).

\section{Estratégia de busca e identificação dos estudos}

A estratégia de busca pode ser entendida como um conjunto de palavras ou termos que são usados em uma base de dados para identificar artigos que têm potencial para responder à pergunta que motivou a revisão (Aromataris \& Munn 2020a). A estratégia de busca elaborada deve ser objetiva e mais completa possível, inclusive em relação ao idioma e período de 
publicação dos artigos selecionados. Além disso, os termos utilizados na busca devem estar claramente delineados e devidamente registrados para garantir a reprodutibilidade da pesquisa (Buehler et al., 2012).

O (JBI) recomenda, em todos os tipos de revisão, uma busca em três etapas (Aromataris \& Munn 2020b). Na primeira etapa, os pesquisadores realizam a busca nas bases de dados. Para isso, eles devem definir os termos de busca controlados (descritores), combinando-os com a pesquisa em linguagem natural (texto livre) e os operadores booleanos (AND, OR e NOT) (Aromataris \& Munn 2020a). Os descritores trazem uniformidade para a pesquisa, criando consistência e precisão, permitindo a triagem dos estudos independente da terminologia usada pelos autores. Já os operadores booleanos, permitem combinar esses termos com o intuito de encontrar artigos que contemplem informações acerca da pergunta formulada (Donato \& Donato, 2019). A segunda etapa busca estudos adicionais analisando a lista de referências dos estudos relevantes, e a terceira etapa é uma busca manual em revistas pertinentes e literatura cinzenta (Aromataris \& Munn 2020a). Além disso, preconiza-se que seja realizada uma busca na produção científica de um pesquisador reconhecidamente referência no tema referente à revisão sistemática. Em qualquer caso, deve ser claramente indicado qual grupo de fontes será examinado (Donato \& Donato, 2019).

A busca de estudos publicados ocorre em bases de dados eletrônicas e as principais na área da saúde são: Medline (Pubmed), Lilacs, Embase, CENTRAL e CINAHL. A qualidade da busca na literatura é conferida a partir de itens elementares como a estratégia de amostragem, tipo de estudo, condutas, amplitude de anos, limites, inclusões e exclusões, termos usados e fontes eletrônicas (Barbosa et al., 2019; Aromataris \& Munn 2020a; Donato \& Donato, 2019).

\section{Seleção dos estudos}

A seleção dos estudos deve ser realizada por pelo menos dois revisores, de maneira independente e cegada, obedecendo aos critérios de elegibilidade. Primeiramente, os dois revisores realizam a leitura de título e resumo de todos os artigos identificados. As discordâncias são verificadas e resolvidas em consenso ou por um terceiro revisor. Em seguida, é realizada a leitura integral dos artigos que preencheram os critérios de elegibilidade, de forma independente, para confirmação da inclusão dos mesmos na revisão. Cabe ressaltar que a triagem dos estudos gera um número muito maior de artigos do que os que realmente serão elegíveis pelos critérios estabelecidos. Entretanto, com a leitura do título e resumo é possível descartar uma boa parte dos artigos identificados por estes não se encaixarem nos critérios de elegibilidade (Aromataris \& Munn 2020a; Donato \& Donato, 2019).

\section{Avaliação da qualidade dos estudos}

A relevância da revisão sistemática depende da qualidade de seus estudos individuais, pois quanto maior a qualidade metodológica dos estudos, menor o risco de viés e mais confiável é o resultado da revisão. Há inúmeras ferramentas de avaliação da qualidade dos estudos, sendo que a maioria delas utiliza uma série de critérios que podem ser assinalados como "atendidos", "não atendidos", "pouco claros" e "não aplicáveis". A partir do cumprimento dos critérios de qualidade, os revisores podem ou não incluir os estudos (Aromataris \& Munn 2020a; Donato \& Donato, 2019).

A escolha do instrumento de avaliação usado para mensurar a qualidade dos estudos incluídos deve ser estabelecida com antecedência e acordada entre os revisores antes do início da avaliação. O detalhamento da avaliação da qualidade metodológica dos estudos selecionados deve constar no protocolo de registro. Um mínimo de dois revisores independentes deve avaliar a qualidade dos estudos. As diferenças devem ser resolvidas em consenso ou por um terceiro revisor (Aromataris \& Munn 2020a; Donato \& Donato, 2019).

\section{Extração de dados}

Os achados dos artigos primários devem ser analisados e extraídos para posterior condução da sistematização e metanálise, se aplicável (Aromataris \& Munn 2020a; Donato \& Donato, 2019). Essa análise visa organizar, categorizar e 
combinar os dados dos estudos incluídos para responder à pergunta de pesquisa. Para isso, recomenda-se utilizar um formulário de extração padronizado. Essa etapa também deve ser realizada por uma dupla de revisores, a fim de evitar tendenciosidades.

É importante salientar que a coleta dos dados pode ser fonte de viés devido ao erro na transcrição ou na coleta de informações relevantes para responder à pergunta da revisão. Somando-se a isso, o próprio processo de extração, pode ser influenciado pela subjetividade e interpretação do revisor. Sendo assim, se houver discrepâncias, elas devem ser resolvidas em consenso ou por um terceiro revisor (Aromataris \& Munn 2020a; Donato \& Donato, 2019).

Outro ponto a ser observado é se a revisão sistemática conduzida abrange estudos quantitativos e se os resultados quantitativos provenientes dos estudos incluídos permitem a realização da metanálise. Caso seja possível realizar metanálise, os pesquisadores também deverão extrair dados para a realização da análise estatística dos resultados apresentados nos estudos primários (Borenstein, Hedges, Higgins \& Rothstein, 2009).

\section{Análise dos resultados}

A priori, todas as revisões sistemáticas que contemplam estudos quantitativos reúnem os achados destes estudos por meio da síntese qualitativa. Esta síntese objetiva produzir a melhor evidência científica disponível sobre um tema específico. Acrescentando-se a isso, também é possível conduzir uma metanálise nos casos em que os estudos primários apresentam homogeneidade nas intervenções avaliadas, ou seja, as características dos estudos incluídos são similares e as variáveis dos estudos estão conceituadas e avaliadas da mesma forma. Este método estatístico é amplamente utilizado na prática baseada em evidências, pois permite avaliar o efeito combinado de vários estudos para uma mesma intervenção (Borenstein et al., 2009; Perera \& Heneghan, 2008). Ademais, vale destacar que resultados qualitativos de diferentes estudos podem ser transformados em dados passíveis de análise estatística por meio da técnica de metassumarização. Essa técnica reúne resultados qualitativos apresentados de modo quantitativamente orientados, na forma de tópicos ou sumários temáticos de surveys ou dados, como a soma de partes dos resultados sobre determinado tema (Lopes \& Fracolli, 2008).

Para sintetizar e analisar os achados de estudos qualitativos, existem várias metodologias. Apesar de elas terem muitas semelhanças entre si, há diferenças claras de abordagem, muitas das quais podem ser explicadas levando-se em consideração os pressupostos epistemológicos de cada metodologia (Barnett-Page \& Thomas, 2009; Tong, Flemming, Mclnnes, Oliver \& Craig, 2012). As metodologias utilizadas são geralmente influenciadas pela pergunta de pesquisa, pelo resultado pretendido no desenvolvimento da síntese, pela posição filosófica do revisor, pelo contexto e pelo público-alvo (Tong et al., 2012). As principais metodologias para conduzir uma síntese de estudos qualitativos incluem: síntese interpretativa crítica, síntese de teoria fundamentada, metaetnografia, metaestudo, síntese temática, síntese de metanarrativa, síntese de narrativa textual, síntese de abordagem estruturada (framework) e "ecological triangulation" (Barnett-Page \& Thomas, 2009). Dessa forma, antes de iniciar uma revisão sistemática de estudos qualitativos, o pesquisador precisa definir qual metodologia irá utilizar, uma vez que esta guiará o decorrer de toda a pesquisa.

\section{Etapas preconizadas para conduzir uma revisão de escopo}

É um método de revisão de literatura cuja proposta é a realização de um mapeamento bibliográfico num determinado campo de interesse, sobretudo quando revisões acerca do tema ainda não foram publicadas (Cordeiro \& Soares, 2019). Tem como objetivos mapear os principais conceitos que apoiam determinada área de conhecimento, examinar a extensão, alcance e natureza da investigação, sumarizar e divulgar os dados da investigação e identificar as lacunas de pesquisas existentes (Arksey \& O'Malley, 2005). Inicialmente, Arksey e O'Malley (2005), propuseram uma metodologia para a realização deste 
tipo de revisão. Contudo, essa estrutura foi aperfeiçoada por Levac, Colquhoun e O'Brien (2010) e posteriormente por Peters e Godfrey (2015), conforme a Figura 1.

Figura 1: Evolução da metodologia sugerida para a elaboração de uma revisão de escopo com seus respectivos autores.

\section{Estrutura de Arksey e O'Malley}

(2005. p. 22-23)

\begin{tabular}{ll}
\hline 1. & Identificar a questão de pesquisa \\
\hline 2. & Identificar estudos relevantes
\end{tabular}

\begin{tabular}{ll}
\hline 3. & Selecionar os estudos \\
\hline 4. & Traçar os dados \\
\hline 5. & $\begin{array}{l}\text { Coletar, resumir e relatar os } \\
\text { resultados }\end{array}$ \\
\hline
\end{tabular}

Melhorias propostas por Levac, Colquhoun e O'Brien (2010. p. 4-8)

*Melhorias propostas por Peters e Godfrey (2015)

Esclarecer e vincular o objetivo e a Definir e alinhar o (s) objetivo (s) e as questão da pesquisa

Equilibrar a viabilidade com a amplitude e abrangência do processo de definição do escopo

Usar uma abordagem de equipe iterativa para selecionar estudos e extrair dados

Incorporar um resumo numérico e uma análise temática qualitativa

Identificar as implicações dos Selecionar as evidências

resultados do estudo para a política, prática ou pesquisa

Adotar a consulta como um Extrair as evidências componente obrigatório da metodologia de estudo de escopo

\section{7.}

8.

9.

perguntas (s)

Desenvolver e alinhar os critérios de inclusão com o (s) objetivo (s) e as perguntas (s)

Descrever a abordagem planejada para busca de evidências, seleção, extração de dados e apresentação das evidências Procurar pelas evidências

Analisar das evidências

Apresentar dos resultados

Resumir as evidências em relação ao objetivo da revisão, tirar conclusões e observar quaisquer implicações dos resultados

Fonte: Adaptado de Peters MD, Godfrey C, McInerney P, Munn Z, Tricco AC, Khalil, H. (2020).

As mudanças observadas acima trouxeram mais rigor para a metodologia da RE e sustentam a abordagem do JBI para a elaboração da mesma (Peters et al., 2020).

Definição da pergunta de pesquisa

A pergunta de pesquisa da RE, assim como na RS, também deve ser formulada de maneira clara e objetiva, pois ela irá direcionar a condução adequada da revisão. Ela também é estruturada a partir dos elementos de um acrônimo, o PCC, que é o mnemônico recomendado para revisões de escopo. PCC significa população, conceito e contexto. Não há necessidade de resultados, intervenções ou fenômenos de interesse explícitos a serem declarados em uma RE (Peters et al., 2020).

Definição dos critérios de elegibilidade

São os critérios que os estudos originais devem atender para serem elegíveis para a revisão, eles devem se enquadrar no acrônimo e na pergunta norteadora. Contudo, como a RE é mais abrangente do que a RS, os critérios de elegibilidade dos seus estudos são menos restritivos (Peters et al., 2020). 
Estratégia de pesquisa e identificação dos estudos

A estratégia de pesquisa deve ser descrita de maneira abrangente, contendo detalhadamente a estratégia de busca utilizada nos bancos de dados e se outras fontes de dados forem utilizadas, as mesmas devem ser anexadas na revisão (Peters $e t$ al., 2020). Deve-se anexar um documento contendo todas as estratégias de pesquisa individuais utilizadas para cada banco de dados pesquisado (Peters et al., 2020). Esse documento valida a qualidade científica da revisão. A RE deve considerar estudos primários, artigos, revisões, literatura cinzenta, tempo de busca apropriado e idioma (Peters et al., 2020). Conforme as recomendações do manual JBI, a triagem dos estudos em uma RE segue as mesmas etapas da triagem na RS. A primeira etapa é uma busca nas bases de dados. Para isso, os pesquisadores devem definir os termos de busca controlados (descritores), combinando-os com a pesquisa em linguagem natural (texto livre) e os operadores booleanos (AND, OR e NOT). A segunda etapa é uma busca de estudos adicionais analisando a lista de referências dos estudos relevantes, e a terceira etapa é uma busca manual em revistas pertinentes e literatura cinzenta.

\section{Seleção dos estudos}

A seleção dos estudos na RE, assim como na RS, é realizada por pelo menos dois revisores, de forma independente e cegada, obedecendo os critérios de elegibilidade pré-definidos no protocolo da revisão. Quaisquer divergências são resolvidas em consenso ou pela decisão de um terceiro revisor. Os estudos são selecionados com base na leitura de título e resumo, seguido da leitura do manuscrito completo (Peters et al., 2020).

Avaliação da qualidade dos estudos

Não é obrigatória em uma RE.

Extração de dados

Esse processo fornece ao leitor uma orientação lógica e descritiva dos resultados que se alinham com os objetivos e as perguntas da RE. Dados que forneçam um resumo dos estudos inseridos também devem ser coletados, como nome do autor, ano da publicação, objetivos, descrição dos participantes, principais conceitos do tema em questão e o contexto no qual ele é inserido. Os dados extraídos devem ser registrados em um formulário de extração padronizado, podendo ser atualizado continuamente. Esta etapa deve ser realizada por uma dupla de revisores, e as discordâncias deverão ser resolvidas por consenso ou pela decisão de um terceiro revisor (Peters et al., 2020).

\section{Análise dos resultados}

Por vezes, os resultados de uma RE são sintetizados de forma mais descritiva, ao invés de analítica, uma vez que o foco desse tipo de revisão é mapear os achados. Frequentemente, contagens simples de frequência serão suficientes para sintetizar resultados quantitativos. É improvável que uma RE necessite de metanálise ou análise qualitativa interpretativa (metassíntese), visto que a RE não tende a avaliar a certeza dos seus resultados. A maneira que os dados serão analisados depende do propósito da revisão e do julgamento do autor (Peters et al., 2020).

\section{Apresentação dos resultados}

Os resultados podem ser apresentados em forma de gráfico, tabela ou em formato descritivo, alinhando os resultados com os objetivos e a pergunta da revisão. Os critérios de inclusão seguindo o PCC podem auxiliar os autores a organizar os resultados de maneira mais adequada. Os resultados apresentados em forma de gráfico ou tabela devem ser acompanhados de um resumo explicativo. Além disso, os resultados podem ser classificados por categoria conceitual, como tipo de amostra, 
população, metodologia adotada, entre outros, e cada categoria citada deve conter uma explicação entendível (Peters et al., 2020).

\section{PRISMA para RE}

Em 2018, a declaração PRISMA para revisões sistemáticas foi estendida para revisões de escopo, originando a Extensão PRISMA para Revisões de Escopo, PRISMA-ScR, (Tricco et al., 2018), fornecendo um checklist de 22 itens para a elaboração deste tipo revisão, apresentado no anexo 2.

\section{Comparação entre revisão sistemática e revisão de escopo}

Os autores do presente estudo compararam as semelhanças e diferenças entre as etapas preconizadas para conduzir uma revisão sistemática ou de escopo por meio dos itens dos checklists PRISMA (Liberati et al., 2009) e PRISMA-ScR (Tricco et al., 2018), respectivamente, conforme a Figura 2.

Figura 2: Comparação entre os critérios de avaliação extraídos dos checklists PRISMA e PRISMA- ScR.

\section{Critérios de avaliação extraídos dos checklists}

PRISMA e PRISMA-ScR

\section{Revisão sistemática}

Revisão de escopo

\section{Título}

Identificar qual o tipo de revisão.

\section{Resumo}

Apresentar um resumo estruturado, incluindo referencial teórico, objetivos, Não cita: intervenção; avaliação fonte de dados, critérios de elegibilidade, participantes e intervenções, $\checkmark \quad$ do estudo; limitações; e, o número avaliação do estudo e síntese dos métodos, resultados, limitações, conclusões de registro da revisão. e implicações dos achados principais, número de registro da revisão.

\section{Introdução}

Descrever a justificativa da revisão no contexto do que já é conhecido.

\section{Objetivos}

Apontar claramente os tópicos abordados com menção ao delineamento do estudo (PICO e demais elementos usados para conceituar as questões e/ou objetivos da revisão).

\section{Métodos}

Protocolo e registro: Indicar onde o protocolo pode ser acessado (por exemplo, um endereço da Web); e se disponível, fornecer informações de registro, incluindo o número de registro.

Critérios de elegibilidade: Especificar as características das fontes de evidência usadas como critérios de elegibilidade (ex.: PICO, anos considerados, idioma, status de publicação).

Fontes de informação: Descrever todas as fontes de informação na pesquisa (ex.: bases de dados com datas de cobertura e contato com autores para

$\checkmark \sqrt{ }$


identificar fontes adicionais), bem como a data em que a pesquisa mais recente foi executada.

Busca: Apresentar a estratégia de busca eletrônica completa para pelo menos um banco de dados, incluindo limites utilizados, de modo que possa ser repetida.

Seleção de estudos: Declarar o processo de seleção dos estudos, ou seja, triagem e elegibilidade, incluídos na revisão, e, se aplicável incluídos na metanálise.

Processo de coleta de dados: Descrever os métodos de extração de dados das fontes de evidência (ex.: formulário piloto, de forma independente, em duplicata) e quaisquer processos para obtenção de dados.

Lista de dados: Listar e definir todas as variáveis para as quais os dados foram buscados (PICOS, fontes de financiamento) e quaisquer suposições e simplificações feitas.

Risco de viés em cada estudo: Descrever os métodos usados para avaliar o risco de viés em cada estudo, especificando se foi feito no nível dos estudos ou resultados, e como essa informação foi usada na análise de dados.

Medidas de sumarização: Definir as principais medidas de sumarização dos resultados (ex.: risco relativo, diferença média).

Síntese dos resultados: Descrever os métodos de tratamento e resumo dos dados que foram coletados, além de incluir medidas de consistência para cada metanálise.

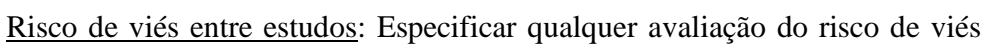
que possa influenciar a evidência cumulativa (ex.: viés de publicação, relato seletivo nos estudos).

Análises adicionais: Descrever métodos de análise adicional (ex.: análise de sensibilidade ou análise de subgrupos, metarregressão), se realizados, indicando quais foram pré-especificados.
Envolve ou não metanálise.

$$
\checkmark
$$

Se apropriado.

Envolve ou não metanálise.

$\begin{array}{cc}\checkmark & \mathrm{X} \\ \text { Envolve ou não } & \text { Normalmente não envolve } \\ \text { metanálise. } & \text { metanálise. }\end{array}$

Normalmente não envolve metanálise.

$\mathrm{X}$

\section{Resultados}

Seleção dos estudos: Fornecer número dos estudos selecionados, avaliados para elegibilidade e incluídos na revisão, com razões para exclusões em cada estágio, de preferência usando um gráfico de fluxo.

Características dos estudos: Para cada estudo, apresentar características para extração de dados e apresentar citações.

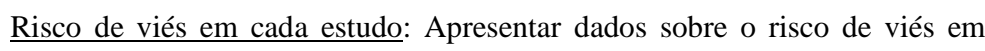
cada estudo e, se disponível, alguma avaliação em resultados.

$\checkmark$


Resultados de estudos individuais: Para todos os desfechos considerados, benefícios ou riscos, apresentar para cada estudo: sumário simples de dados para cada grupo de internação, bem como os efeitos estimados e intervalos de confiança, preferencialmente por meio de gráficos de floresta.

Síntese de resultados: Apresentar resultados para cada metanálise feita, incluindo intervalos de confiança e medidas de consistência.

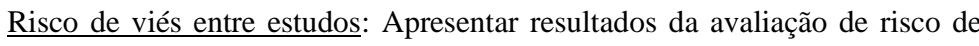
viés entre os estudos

Análises adicionais: Apresentar resultados de análises adicionais, se realizadas (ex.: análise de sensibilidade ou subgrupos, metarregressão).

Para cada fonte de evidência incluída, apresentar os dados relevantes que foram mapeados e

que se relacionam com as questões e os objetivos da revisão.

Apresentar os resultados em forma de gráfico, conforme eles estão relacionados às questões e objetivos da revisão.

$\mathrm{X}$

$\mathrm{X}$

\section{Discussão}

Sumário de evidência: Resumir os principais resultados, incluindo a força de evidência para cada resultado e considerar a relevância para grupos-chave.

Limitações: Discutir as limitações no nível dos estudos e dos desfechos (ex.: risco de viés) e no nível da revisão (ex.: obtenção incompleta de pesquisas identificadas, viés de relato). da revisão

Conclusões: Apresentar a interpretação geral dos resultados no contexto de outras evidências e implicações para futuras pesquisas.

\section{Financiamento}

Descrever as fontes de financiamento para a revisão e outros suportes e o papel dos financiadores na revisão.

X: Não se aplica a revisão de escopo

Fonte: Produzido pelo autor através do Excel.

De acordo com a Figura 2, as revisões se assemelham por seguirem um processo estruturado, mas se diferem em alguns elementos metodológicos, observados por meio da comparação dos itens dos checklists apresentados acima.

A revisão de escopo, diferente da revisão sistemática, não apresenta análises estatísticas por metanálise, medidas de sumarização, avaliação da qualidade dos estudos (avaliação do risco de viés) e outros métodos de análises adicionais. Adicionalmente, as revisões também se diferenciam quanto a sua aplicação, pois devem ser realizadas por motivos diferentes.

As revisões sistemáticas são indicadas para confirmar se práticas atuais são baseadas em evidências, para comprovar a qualidade das evidências, para solucionar possíveis conflitos em evidências que possam estar induzindo a práticas discordantes, identificar lacunas e tendenciosidades nas evidências, para orientar a tomada de decisão e desenvolver políticas. Dessa forma, esse tipo de revisão pode ser considerado o pilar dos cuidados em saúde baseados em evidências, por conseguinte, são amplamente utilizadas no desenvolvimento de diretrizes clínicas confiáveis (Munn et al., 2018). 
Em contrapartida, as revisões de escopo são indicadas para mapear as evidências disponíveis, para indicar o escopo ou a dimensão da literatura existente sobre um determinado tema, esclarecer conceitos, identificar e analisar lacunas no conhecimento, fornecer uma visão geral do foco dos estudos encontrados, investigar evidências em ascensão quando dados mais específicos ainda não estão disponíveis, servindo como base para uma futura revisão sistemática, ou ainda, pode ser realizada em casos em que a revisão sistemática seria incapaz de atender aos objetivos ou requisitos necessários para a elaboração do estudo (Munn et al., 2018). Logo, não se trata de buscar a melhor evidência, mas de reunir os vários tipos de evidências e mostrar como foram produzidas. Também não se destina a classificar a força da evidência, mas rastreá-la. Além disso, as revisões de escopo também são apropriadas para examinar estudos para a tomada de decisão no campo teóricometodológico, a partir de mapeamento de teorias e metodologias que se destinam a informar os pesquisadores (Cordeiro \& Soares, 2019., Peters et al., 2020).

\section{Etapa 2 - Comparação e análise do uso de revisões de escopo e sistemática na área do cuidado ao paciente na farmácia}

Foram selecionados dois artigos, um de revisão sistemática e outro de escopo, que abordam a descrição de ferramentas desenvolvidas para a seleção de pacientes para serviços clínicos farmacêuticos. A priorização desses pacientes ocorre em função da escassez de recursos e da alta demanda por serviços de saúde. O artigo "Patient prioritization for pharmaceutical care in hospital: A systematic review of assessment tools" (Alshakrah, Steinke \& Lewis, 2019) descreve as ferramentas de avaliação existentes usadas por farmácias hospitalares para avaliar a prioridade e/ou complexidade do paciente para ser elegível nos serviços clínicos em Farmácia. Em contrapartida, o artigo "Tools to prioritize outpatients for pharmaceutical service: A scoping review" (Sousa et al., 2020) retrata as ferramentas para a seleção de pacientes para atendimento clínico farmacêutico prestado em serviços de atenção à saúde primária e ambulatorial. Os autores do presente estudo avaliaram se as revisões selecionadas atenderam os itens dos checklist dos respectivos tipos de revisão, tal análise e comparação das revisões pode ser observada na Figura 3.

Figura 3: Comparação entre revisão sistemática e revisão de escopo na área de cuidado ao paciente na Farmácia.

\begin{tabular}{|c|c|c|}
\hline Itens analisados & REVISÃO SISTEMÁTICA & REVISÃO DE ESCOPO \\
\hline Título do artigo & $\begin{array}{l}\text { Priorização de pacientes para assistência farmacêutica } \\
\text { em hospitais: uma revisão sistemática das ferramentas } \\
\text { de avaliação }\end{array}$ & $\begin{array}{l}\text { Ferramentas para priorizar pacientes } \\
\text { ambulatoriais para serviços farmacêuticos: uma } \\
\text { revisão de escopo }\end{array}$ \\
\hline Pergunta de pesquisa & $\begin{array}{l}\text { *Quais as ferramentas para priorizar pacientes com } \\
\text { maior risco de Problemas Relacionados aos } \\
\text { Medicamentos (PRM) para serviço clínico } \\
\text { farmacêutico em ambiente hospitalar? Como essas } \\
\text { ferramentas funcionam? }\end{array}$ & $\begin{array}{l}\text { *Quais as ferramentas desenvolvidas para } \\
\text { selecionar pacientes para serviços clínicos } \\
\text { farmacêuticos prestados em ambulatórios e na } \\
\text { atenção primária? Como essas ferramentas } \\
\text { funcionam? }\end{array}$ \\
\hline Número de artigos identificados & 14.937 & 5.501 \\
\hline Número de artigos incluídos & 19 & 13 \\
\hline Objetivo do estudo & $\begin{array}{l}\text { Fornecer uma visão geral e descrever as ferramentas de } \\
\text { avaliação existentes com foco na qualidade do estudo, } \\
\text { temas, validade da ferramenta, fatores de risco e classes } \\
\text { de medicamentos de alto risco }\end{array}$ & $\begin{array}{l}\text { Mapear e descrever ferramentas desenvolvidas } \\
\text { para a seleção de pacientes para serviços } \\
\text { farmacêuticos prestados na atenção primária à } \\
\text { saúde e em ambientes ambulatoriais }\end{array}$ \\
\hline Revista e ano de publicação & Research in Social and Administrative Pharmacy, 2019 & $\begin{array}{l}\text { Research in Social and Administrative } \\
\text { Pharmacy, } 2020\end{array}$ \\
\hline
\end{tabular}




\section{Estratégia de Busca}

Base de Dados

Detalhamento metodológico
1 Prioridade ou triagem ou acuidade ou complexo.

2. Ferramenta ou pontuação ou tela ou critérios, escala, classificação, avaliação, avaliação clínica, ferramenta, instrumento, medida, estratificação, software.

3. Hospital, atenção secundária.

4. Farmácia, farmacêutico, farmacêutico, serviço de farmácia, farmácia hospitalar, farmácia clínica, serviço farmacêutico clínico.
1. Serviços farmacêuticos ou serviços de farmácia ou assistência farmacêutica ou cuidados, produtos farmacêuticos ou community pharmacy services ou pharmaceutical service, community ou serviço, farmacêutico comunitário ou serviço de farmácia comunitária ou community pharmaceutical services ou medication therapy management ou gestão de terapia com medicação ou gerenciamento da terapia medicamentosa ou melhoria dos medicamentos prescritos. 2. Triagem ou seleção de paciente ou recrutamento de assunto de pesquisa ou seleção de assunto de pesquisa ou seleção de tratamento ou seleção de assuntos ou recrutamento de paciente ou critérios de seleção. 3. Avaliação de risco ou avaliação de risco à saúde ou avaliação de risco benefício ou avaliação de benefício risco, ajuste de risco benefício, ajuste de risco. 4. Instrumento ou avaliação ou classificação ou avaliação clínica ou ferramenta de avaliação ou priorização. 5 . Ambulatory care ou care outpatient ou health services, outpatient ou serviço de saúde, ambulatório ou serviço, saúde ambulatorial ou serviços ambulatoriais ou serviço, paciente ambulatorial ou serviços, saúde ambulatorial ou cuidado urgente ou cuidados, urgente ou visitas à clínica. 6 . Atenção primária à saúde ou atenção à saúde, primária ou cuidados de saúde, primário ou cuidados de saúde, primário. 7. Pacientes ambulatoriais ou pacientes externos ou ambulatorial.

Medline, Embase, Cochrane Central Register of Medline, Embase, International Pharmaceutical Controlled Trials (CENTRAL) e Latin Abstracts, Scopus e Web of Science.

American and Caribbean Health Sciences (LILACS).

Triagem dos estudos, exclusão das duplicatas, seleção dos estudos por um único revisor, resolução das divergências da seleção por uma equipe de revisores, extração dos dados por dupla de revisores, resolução das divergências da extração por dupla de revisores, avaliação da qualidade dos estudos por um único revisor e análise dos resultados.

Triagem dos estudos, exclusão das duplicatas,
seleção dos estudos por uma equipe de
revisores, resolução das divergências da seleção
pela equipe de revisores, extração dos dados
por uma dupla de revisores, resolução das
divergências da extração por um terceiro
revisor, análise dos resultados.




\begin{tabular}{|c|c|c|}
\hline $\begin{array}{l}\text { Metodologia dos estudos } \\
\text { incluídos }\end{array}$ & Quantitativa, qualitativa e mista. & $\begin{array}{l}\text { Ensaios clínicos randomizados, experimentais e } \\
\text { observacionais. }\end{array}$ \\
\hline $\begin{array}{l}\text { Instrumento de avaliação dos } \\
\text { estudos incluídos }\end{array}$ & $* *$ Hawker & Não avaliou a qualidade dos estudos $* * *$ \\
\hline Tratamento dos dados & Metassumarização* & Metassumarização* \\
\hline
\end{tabular}

* Interpretação do autor deste estudo. **Ferramenta de avaliação da qualidade de estudos que inclui 9 questões com 4 critérios: bom, regular, ruim e muito ruim. Avalia trabalhos de publicação díspares, considerando qualitativos, quantitativos, artigos de revisão e resumos de conferências. ***Não era o objetivo da revisão.

Fonte: Produzido pelo autor através do Excel.

Após analisar a metodologia das revisões empregadas na área de cuidado ao paciente na Farmácia foi possível verificar que a revisão sistemática "Patient prioritization for pharmaceutical care in hospital: A systematic review of assessment tools" (Alshakrah et al., 2019) não seguiu as orientações da diretriz PRISMA (Liberati et al., 2009) para a elaboração deste tipo de revisão, referência apresentada pelo autor do artigo como guia para a condução da mesma, nem as orientações do manual JBI para elaboração de revisões sistemáticas (Aromataris \& Munn 2020a). Isso porque, tanto a seleção dos estudos quanto a avaliação da qualidade dos estudos foram conduzidas por um único revisor, e não por uma dupla de revisores como indica os guias citados, o que pode levar a tendenciosidades na seleção dos estudos e resultados não significativos.

Além disso, o objetivo do estudo (Alshakrah et al., 2019) apontada pelo autor como sendo "Fornecer uma visão geral estruturada e descrever as ferramentas de avaliação existentes com foco na qualidade do estudo, temas, validade da ferramenta, fatores de risco e classes de medicamentos de alto risco" não condiz com a aplicação de uma revisão sistemática, mas sim com uma revisão de escopo, pois o estudo visa apresentar e descrever as ferramentas de priorização de pacientes, e não, avaliar quais ferramentas são mais eficazes para selecionar esses pacientes.

Em contrapartida, a revisão de escopo analisada "Tools to prioritize outpatients for pharmaceutical service: A scoping review" (Sousa et al., 2020) seguiu todas as orientações da diretriz PRISMA-ScR (Tricco et al., 2018) e do manual JBI para elaboração de revisões de escopo (Peters et al., 2020).

Dada a importância das revisões de literatura como instrumento para a síntese de informação e guia para tomada de decisão baseada em evidência, requer mais minuciosidade dos autores durante a elaboração das mesmas, sendo mais rigorosos quanto às especificidades, critérios para elaboração e finalidade de cada revisão.

Dessa forma, se o pesquisador deseja buscar evidências para desenvolver diretrizes e políticas confiáveis, orientar a prática clínica, avaliar a eficácia de um tratamento ou confirmar se a prática atual é baseada em evidências relevantes, a revisão sistemática é a metodologia adequada. Por outro lado, se a finalidade for mapear as evidências disponíveis, estudar temas em ascensão, identificar lacunas no conhecimento ou discutir concepções, o pesquisador deve optar por uma revisão de escopo.

\section{Conclusão}

Embora as revisões se destinem a objetivos diferentes, ambas apresentam uma metodologia criteriosa, mas se diferem quanto a alguns elementos metodológicos, como, por exemplo, avaliação crítica da qualidade dos estudos e tratamento de dados mais elaborados presentes na revisão sistemática. A associação correta entre a questão de pesquisa e a escolha da revisão é crucial para a sua condução, uma vez que, para cada tipo de revisão há uma orientação metodológica e uma indicação de uso. 
Dada a importância das revisões de literatura como instrumento para a síntese de informação e guia para tomada de decisão baseada em evidência, requer mais minuciosidade dos autores durante a elaboração das mesmas, sendo mais rigorosos quanto às especificidades, critérios para elaboração e finalidade de cada revisão.

Dessa forma, se o pesquisador deseja buscar evidências para desenvolver diretrizes, orientar a prática clínica, avaliar a eficácia de um tratamento ou confirmar se a prática atual é baseada em evidências relevantes, a revisão sistemática é a metodologia adequada. Por outro lado, se a finalidade for identificar conceitos e descrições em artigos científicos, fornecer uma visão geral das evidências existentes, estudar temas em ascensão, identificar lacunas no conhecimento ou discutir concepções, o pesquisador deve optar por uma revisão de escopo.

\section{Referências}

Alshakrah, M. A., Steinke, D. T., \& Lewis, P. J. (2019). Patient prioritization for pharmaceutical care in hospital: a systematic review of assessment tools. Research in Social and Administrative Pharmacy, 15(6), 767-779.

Arksey, H., \& O'Malley, L. (2005). Scoping studies: towards a methodological framework. International journal of social research methodology, 8(1), 19-32.

Aromataris, E. M. Z. E., \& Munn, Z. (2020a). Chapter 1: JBI Systematic Reviews. Institute reviewer's manual.

Aromataris, E. M. Z. E., \& Munn, Z. (2020b). JBI manual for evidence synthesis. Institute reviewer's manual.

Barbosa, F.T., Lira, A.B., Neto, O.B., Santos, L.L., Santos, I.O., Barbosa, L.T., Ribeiro, M.V.M.R., \& Sousa-Rodrigues, C.F. (2019). Tutorial para execução de revisões sistemáticas e metanálises com estudos de intervenção em anestesia. Revista Brasileira de Anestesiologia, 69, $299-306$.

Barnett-Page, E., \& Thomas, J. (2009). Methods for the synthesis of qualitative research: a critical review. BMC medical research methodology, 9(1), 1-11.

Biruel, E. P., \& Pinto, R. (2011). Bibliotecário um profissional a serviço da pesquisa. Anais do XXIV Congresso Brasileiro de Biblioteconomia, Documentação e Ciência da Informação. Maceió, Alagoas, Brasil.

Borenstein, M., Hedges, L. V., Higgins, J. P.T., \& Rothstein, H. R. (2009). Introduction to meta-analysis. (1ª ed). Chichester, U.K: John Wiley \& Sons.

Buehler, A.M., Figueiró, M.F., Cavalcanti, A.B., \& Berwanger, O. (2012). Diretrizes Metodológicas: Elaboração de revisão sistemática e metanálise de ensaios clínicos randomizados. $\left(1^{\mathrm{a}} \mathrm{ed}\right)$. Brasília: MS.

Buehler, A.M., Figueiró, M.F., Cavalcanti, A.B., \& Berwanger, O. (2014). Diretrizes Metodológicas: Elaboração de revisão sistemática e metanálise de estudos de acurácia diagnóstica. (1ª ed). Brasília: MS.

Comissão de Farmácia Comunitária do CRF-PR. (2015). Prática farmacêutica: saúde baseada em evidências. Recuperado em 10 dezembro, 2019, de https://crf-pr.org.br/site/noticia/visualizar/id/6318/Artigo---Pratica-farmaceutica-saude-baseada-em-evidencias

Cordeiro, A. M., Oliveira, G. M. D., Rentería, J. M., \& Guimarães, C. A. (2007). Revisão sistemática: uma revisão narrativa. Revista do Colégio Brasileiro de Cirurgiões, 34, 428-431.

Cordeiro, L., \& Soares, C. B. (2019). Revisão de escopo: potencialidades para a síntese de metodologias utilizadas em pesquisa primária qualitativa. Boletim do Instituto de Saúde - BIS, 20(2), 37-43.

Donato, H., \& Donato, M. (2019). Etapas na Condução de uma Revisão Sistemática. Acta Médica Portuguesa, 32(3).

Hedges, L. V., \& Cooper, H. (1994). The handbook of research synthesis. (1 $\left.{ }^{\mathrm{a}} \mathrm{ed}\right)$. New York: Russell Sage Foundation.

Köche, J.C. (2011). Fundamentos de metodologia científica: teoria da ciência e iniciação à pesquisa. (29a ed). Petrópolis: Vozes.

Levac, D., Colquhoun, H., \& O'Brien, K. K. (2010). Scoping studies: advancing the methodology. Implementation science, 5(1), 1-9.

Liberati, A., Altman, D.G., Tetzlaff, J., Mulrow, C., Gøtzsche, P.C., Ioannidis, J.P., Clarke, M., Devereaux, P.J., Kleijnen, J., \& Moher, D. (2009). The PRISMA statement for reporting systematic reviews and meta-analyses of studies that evaluate health care interventions: explanation and elaboration. Journal of clinical epidemiology, 62(10), e1-e34.

Lopes, A. L. M., \& Fracolli, L. A. (2008). Revisão sistemática de literatura e metassíntese qualitativa: considerações sobre sua aplicação na pesquisa em enfermagem. Texto \& Contexto-Enfermagem, 17, 771-778.

Mancini, M. C., \& Sampaio, R. F. (2006). Quando o objeto de estudo é a literatura: estudos de revisão. Brazilian Journal of Physical Therapy, 10(4).

Mendes, K. D. S., Silveira, R. C. D. C. P., \& Galvão, C. M. (2008). Revisão integrativa: método de pesquisa para a incorporação de evidências na saúde e na enfermagem. Texto \& contexto-enfermagem, 17, 758-764.

Munn, Z., Peters, M. D., Stern, C., Tufanaru, C., McArthur, A., \& Aromataris, E. (2018). Systematic review or scoping review? Guidance for authors when choosing between a systematic or scoping review approach. BMC medical research methodology, 18(1), 1-7.

Perera, R., \& Heneghan, C. (2008). Interpreting meta-analysis in systematic reviews. BMJ Evidence-Based Medicine, 13(3), 67-69. 
Research, Society and Development, v. 10, n. 12, e08101219915, 2021

(CC BY 4.0) | ISSN 2525-3409 | DOI: http://dx.doi.org/10.33448/rsd-v10i12.19915

Peters, M. D., Godfrey, C. M., Khalil, H., McInerney, P., Parker, D., \& Soares, C. B. (2015). Guidance for conducting systematic scoping reviews. JBI Evidence Implementation, 13(3), 141-146.

Peters, M. D., Godfrey, C., McInerney, P., Munn, Z., Tricco, A. C., \& Khalil, H. (2020). Chapter 11: scoping reviews. Joanna Briggs Institute reviewer's manual.

Sampaio, R. F., \& Mancini, M. C. (2007). Estudos de revisão sistemática: um guia para síntese criteriosa da evidência científica. Brazilian Journal of Physical Therapy, 11, 83-89.

Sousa, M. D. C. V. B., Fernandes, B. D., Foppa, A. A., Almeida, P. H. R. F., Mendonça, S. D. A. M., \& Chemello, C. (2020). Tools to prioritize outpatients for pharmaceutical service: A scoping review. Research in Social and Administrative Pharmacy, 16(12), 1645-1657.

Stern, C., Jordan, Z., \& McArthur, A. (2014). Developing the review question and inclusion criteria. AJN The American Journal of Nursing, 114(4), 53-56.

Suzumura, E.A., Buehler, A.M., Pereira, S.B., Figueiró, M., Oliveira, C., Cavalcanti, A.B., \& Berwanger, O. (2014). Diretrizes metodológicas: Elaboração de revisão sistemática e metanálise de estudos observacionais comparativos sobre fatores de risco e prognóstico. ( $1^{\mathrm{a}}$ ed). Brasília: MS.

Tong, A., Flemming, K., McInnes, E., Oliver, S., \& Craig, J. (2012). Enhancing transparency in reporting the synthesis of qualitative research: ENTREQ. BMC medical research methodology, 12(1), 1-8.

Tricco, A.C., Lillie, E., Zarin, W., O'Brien, K.K., Colquhoun, H., Levac, D., Moher, D., Peters, M.D.J., Horsley, T., Weeks, L., Hempel, S., Akl, E.A., Chang, C., McGowan, J., Stewart, L., Hartling, L., Aldcroft, A., Wilson, M.G., Garritty, C., Lewin, S., Godfrey, C.M., Macdonald, M.T., Langlois, E.V., SoaresWeiser, K., Moriarty, J., Clifford, T., Tunçalp, Ö. \& Straus, S.E. (2018). PRISMA extension for scoping reviews (PRISMA-ScR): checklist and explanation. Annals of internal medicine, 169(7), 467-473. 